\title{
Estudio de la vulnerabilidad sísmica de las viviendas informales en el Asentamiento Humano San Carlos de Murcia, Chachapoyas, 2017
}

\section{Study of the seismal vulnerability of informal housing in the Human Settlement San Carlos de Murcia, Chachapoyas, 2017}

\author{
Rocio Elizabeth López Ramírez
}

\section{RESUMEN}

Esta investigación de tipo descriptivo, tuvo como objetivo determinar el grado de vulnerabilidad sísmica de las viviendas informales en el Asentamiento Humano San Carlos de Murcia. Para ello, fue necesario recoger la información de las viviendas aplicando una ficha de recolección de datos, que luego fue analizada empleando el Método de Evaluación del Grado de Vulnerabilidad Sísmica de la Asociación Colombiana de Ingeniería Sísmica (AIS), siendo los parámetros evaluados: aspectos geométricos, aspectos constructivos, aspectos estructurales, cimentación, suelo y entorno; de una muestra representativa de 30 viviendas. Los resultados mostraron que el $3.33 \%$ de las viviendas estudiadas presentan un grado de vulnerabilidad sísmica baja, el 36.67\% de las viviendas estudiadas presentan un grado de vulnerabilidad sísmica media y el 60\% de las viviendas estudiadas presentan un grado de vulnerabilidad sísmica alta, comprobando así la hipótesis de la investigación.

Palabras clave: Vulnerabilidad sísmica, Viviendas informales.

\begin{abstract}
This descriptive research aimed to determine the degree of seismic vulnerability of informal homes in the San Carlos Human Settlement in Murcia. For this, it was necessary to collect the information of the houses by applying a Data Collection Form, which was then analyzed using the Method of Evaluation of the Degree of Seismic Vulnerability of the Colombian Association of Seismic Engineering (AIS), the parameters being evaluated: aspects geometric, constructive aspects, structural aspects, foundation, soil and environment; of a representative sample of 30 homes. The results is that $3.33 \%$ of the homes studied have a low degree of seismic vulnerability, $36.67 \%$ of the homes studied have a medium degree of seismic vulnerability and $60 \%$ of the homes studied have a high degree of seismic vulnerability, checking thus the hypothesis of the investigation.
\end{abstract}

Keywords: Seismic vulnerability, Informal housing.

\footnotetext{
${ }^{1}$ Universidad Nacional Toribio Rodríguez de Mendoza de Amazonas, Facultad de Ingeniería Civil y Ambiental, Escuela Profesional de Ingeniería Civil.
} 


\section{INTRODUCCIÓN}

El Perú, geográficamente, forma parte del llamado cinturón de fuego del Pacífico lo que le hace una de las regiones de más actividad sísmica en la tierra, por lo tanto, está expuesto a sufrir sismos de baja o alta intensidad.

La ciudad de Chachapoyas, ubicada en la región Amazonas y considerada parte de la zona sísmica $\mathrm{N}^{\circ}$ 02 (Norma E.030, 2016); en los últimos años ha ido creciendo en población, lo que ha aumentado la necesidad de viviendas, sobre todo en los sectores de bajos ingresos quienes han optado en invadir terrenos para construir sus viviendas.

San Carlos de Murcia es uno de estos asentamientos humanos, ubicado en Chachapoyas, que fue invadido hace pocos años; la posibilidad de vivir en aquel lugar hizo que la población construya sus casas de manera económica y breve, dando origen a la autoconstrucción de viviendas.

El AA.HH. San Carlos de Murcia no cuenta con ningún tipo de estudio formal que determine el grado de vulnerabilidad sísmica de sus viviendas, esto constituyó un motivo para iniciar la presente investigación. Para la cual se planteó el siguiente problema: ¿Cuál es el grado de vulnerabilidad sísmica de las viviendas informales del Asentamiento Humano San Carlos de Murcia, Chachapoyas, Amazonas - 2017? Siendo el objetivo el de determinar el grado de vulnerabilidad sísmica de este sector. Este trabajo debió; primero, identificar y seleccionar las viviendas informales según indicadores que evidencien que estas se encuentran en mayor riesgo; segundo, evaluar los patrones generales: aspecto geométrico, aspecto constructivo, aspecto estructural, cimentación, suelo y entorno de las viviendas informales que indica el Método de la Asociación Colombiana de Ingeniería Sísmica; y por último, determinar las viviendas con mayor riesgo de vulnerabilidad y las posibles causas de daño sísmico. La investigación consistió en recoger información de las viviendas informales, mediante una Ficha de Recolección de Datos, para luego tener que ser analizados mediante los parámetros que señala el Método de Evaluación del Grado de Vulnerabilidad Sísmica de la Asociación Colombiana de Ingeniería Sísmica (AIS), y teniendo en cuenta las normas técnicas E.030 Diseño sismorresistente, E.070 Albañilería, E.080 Diseño y construcción con tierra reforzada del Reglamento Nacional de Edificaciones (R.N.E.).

Cada parámetro de las viviendas fue medido en un grado de vulnerabilidad baja, media y alta. Y finalmente se determinó el grado de vulnerabilidad sísmica de todas las viviendas informales del asentamiento humano, resultando ser alto en un $60 \%$.

\section{MATERIAL Y MÉTODOS}

\section{Localización}

La investigación se desarrolló en el AA. HH. San Carlos de Murcia, ubicado en el distrito de Chachapoyas, provincia Chachapoyas, región Amazonas del Perú.

\section{Diseño de la investigación}

La investigación es del tipo descriptivo no experimental. Con un universo muestral de 30 viviendas informales, 17 de adobe y 13 de albañilería; seleccionadas teniendo en cuenta su ubicación y topografía.

\section{Metodología}

Inductivo

\section{Técnicas e instrumentos}

La técnica empleada para el desarrollo de esta investigación, fue la Observación.

Para la obtención de información se utilizó la Ficha de Recolección de Datos. Esta ficha recogió las características de cada vivienda, comprendiendo su información general, terreno y cimentación, aspectos geométricos, aspectos constructivos y estructurales, etc.

\section{Procedimiento}

1. Identificación y selección de las viviendas Fueron seleccionadas 30 viviendas informales para ser evaluadas, teniendo en cuenta las siguientes características: Que éstas se ubiquen a rivera de la quebrada de Murcia por encontrarse, evidentemente, en mayor riesgo y las que se ubiquen en zonas de relieve accidentado (de fuertes pendientes).

Para la identificación de cada una de ellas, se empleó el plano catastral de la ciudad de Chachapoyas.

2. Adaptación de la Ficha de recolección de datos Para emplear el Método de Evaluación del Grado de Vulnerabilidad Sísmica de la Asociación Colombiana de Ingeniería Sísmica (AIS), fue necesario recoger las características de cada vivienda, al carecer el Método del AIS de una Ficha de Recolección de Datos se tuvo que adaptar el Formato de Captura de Datos para Evaluación Estructural de la Red Nacional de Evaluadores (Cárdenas, Flores y López, 2011). El instrumento se validó con un grupo de estudiantes de los últimos ciclos de la carrera de Ingeniería Civil y se sometió a juicio de expertos.

3. Aplicación de la Ficha de recolección de datos Ficha de Recolección de Datos de la vivienda $\mathrm{N}^{\circ} 21$ : 


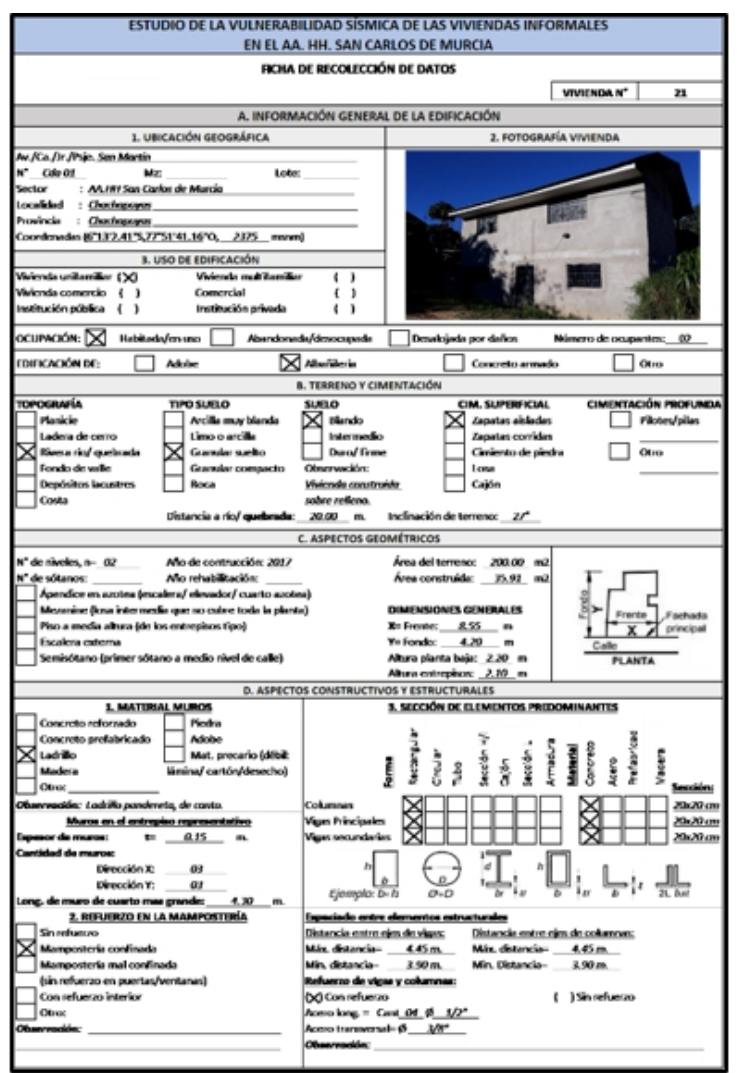

Figura 1. Ficha de Recolección de Datos. (1/3)

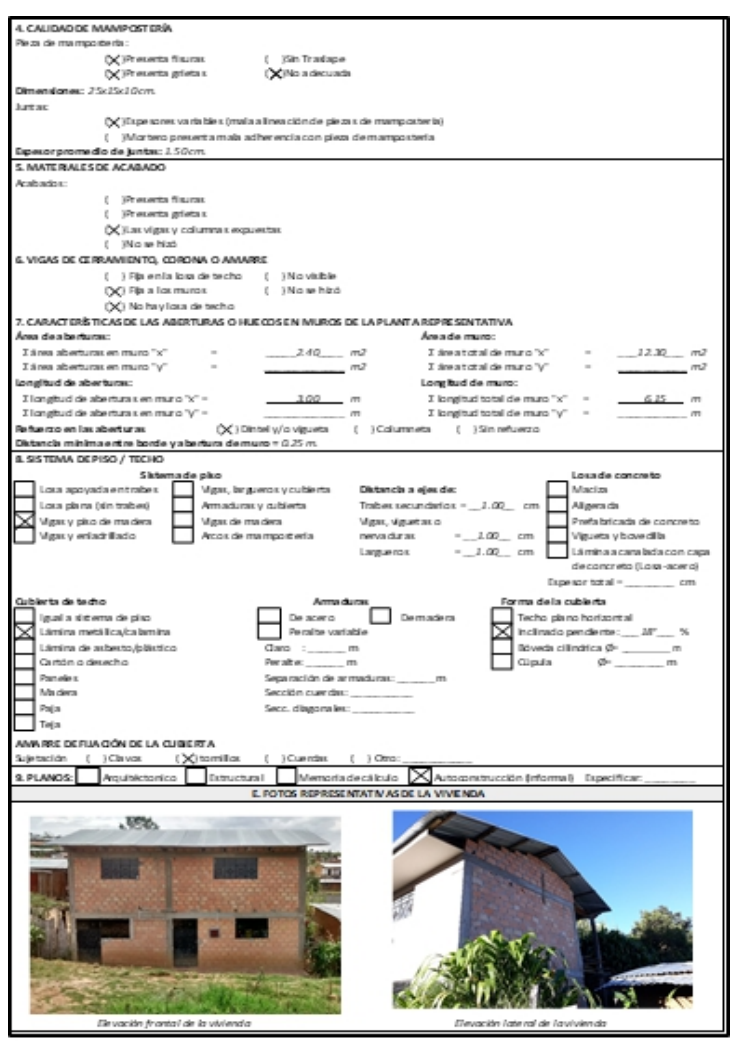

Figura 2. Ficha de Recolección de Datos. (2/3)

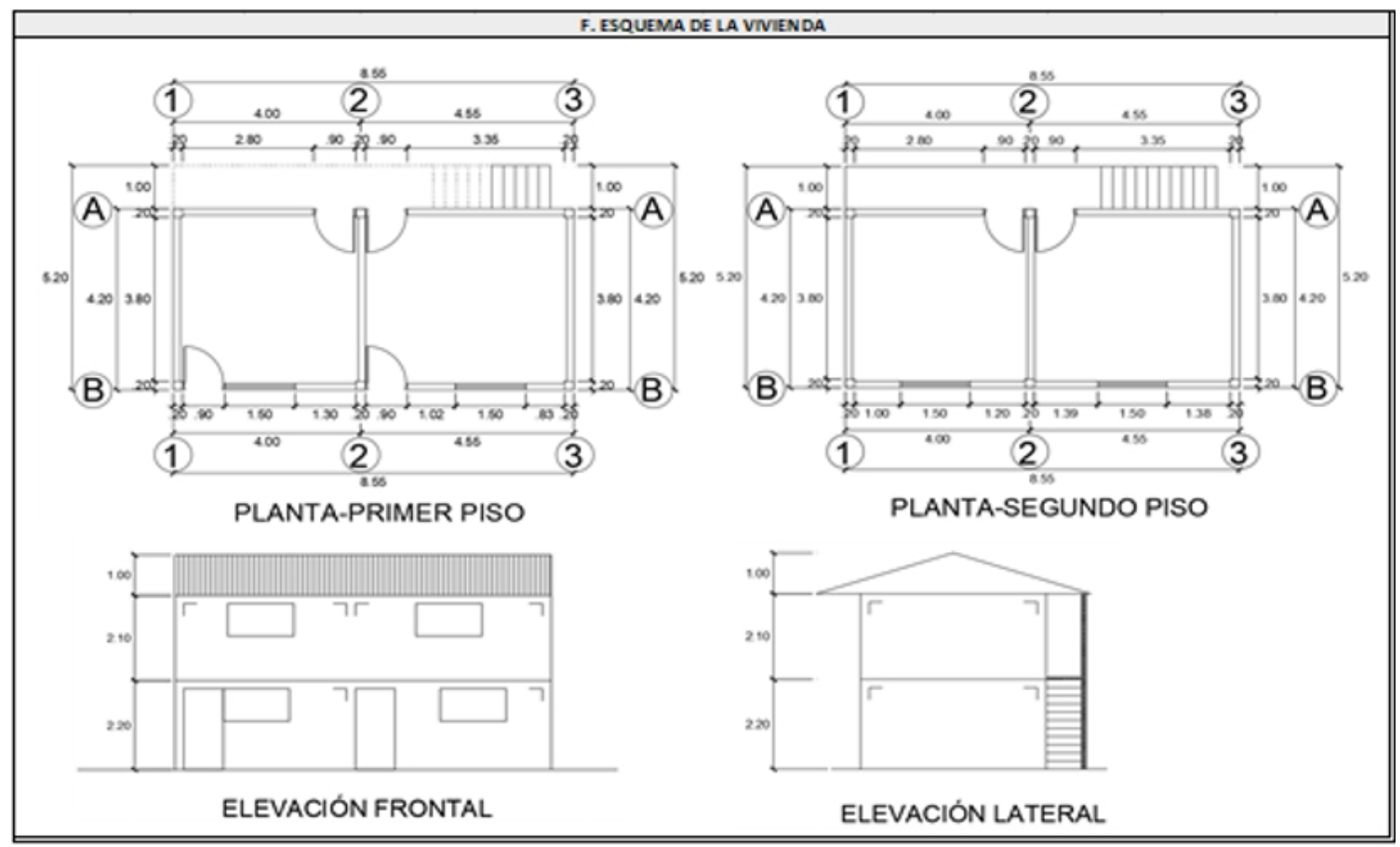

Figura 3. Ficha de Recolección de Datos. (3/3) 


\section{Evaluación de parámetros de investigación}

Se analizó la información de la Ficha de Recolección de Datos y comparó los patrones del Método del AIS.

La calificación del grado de vulnerabilidad se hizo de acuerdo la matriz del Método del AIS (2001).

Esta etapa se describe a continuación:

Parámetro 01:Aspectos Geométricos

A) Irregularidad en planta de la edificación

Dimensiones de la vivienda: $X=8.55 \mathrm{~m}$. y $\mathrm{Y}=4.20 \mathrm{~m}$; el largo viene a ser menor que 3 veces el ancho. Por lo tanto, la vivienda.

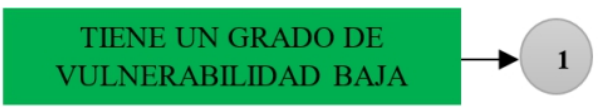

B) Cantidad de muros en las dos direcciones

Se aplica la Norma E.030 del R.N.E., que indica calcular la densidad de muros confinados:

$$
\frac{\text { Área de corte de los muros }}{\text { Área de planta }}=\frac{\sum L . t}{A p} \geq \frac{Z U S N}{56}
$$

Datos de la vivienda de la Ficha de Recolección de Datos y de la Norma E.030:

- Área total techada:

Primery segundo piso: $35.91 \mathrm{~m} 2$

- Sumatoria de longitud por el espesor de los muros confinados ( $\sum$ L.t $)$ :

Primer piso: "X" $=1.12 \mathrm{e}$ "Y" $\mathrm{Y}=1.71$

Segundo piso: " $\mathrm{X} "=1.52 \mathrm{e}$ "Y"=1.71

- Factor de zona $(Z)=0.25($ Zona 2$)$

- Factor de uso de edificación $(\mathrm{U})=1.00$ (Para vivienda)

- Factor de amplitud de suelo (S) = 1.40 (Suelo blando)

- $\operatorname{Númerode~} \operatorname{pisos}(\mathrm{n}) \quad=2.00$ (2 Pisos)

Cálculo de la densidad mínima de muros portantes:

PRIMER PISO:

$$
\begin{aligned}
& \text { Eje "X": } \sum(\text { L.t }) / A p=0.031 \geq 0.013 \\
& \text { Eje "Y": } \sum \text { (L.t) } / A p=0.048 \geq 0.013
\end{aligned}
$$

\section{SEGUNDO PISO:}

$$
\begin{aligned}
& \text { Eje "X": } \sum(\text { L.t }) / A p=0.042 \geq 0.013 \\
& \text { Eje "Y": } \left.\sum \text { (L.t }\right) / A p=0.048 \geq 0.013
\end{aligned}
$$

Tenemos que la densidad de muros en ambas direcciones es mayor que la densidad de muros mínimo requerido por el R.N.E. Por lo tanto, la vivienda.

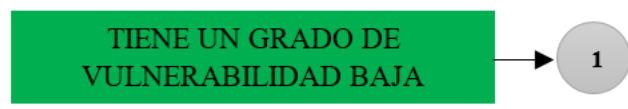

La vivienda cuenta con dos (02) niveles y presenta una regularidad en la altura, desde la cimentación hasta la cubierta. Por lo tanto,

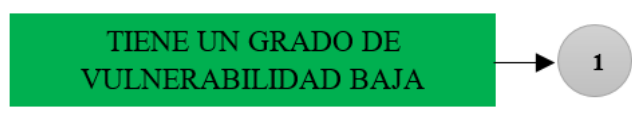

Parámetro 02: Aspectos Constructivos

A) Calidad de las juntas de pega del mortero

El espesor de las juntas de esta vivienda varía provocando una mala alineación de las unidades de ladrillo, siendo el promedio $15 \mathrm{~mm}$ o $1.5 \mathrm{~cm}$. Por lo tanto,

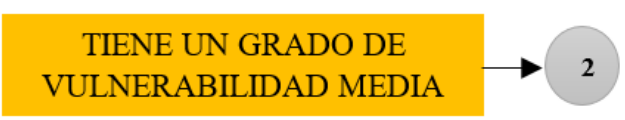

B) Tipo y disposición de las unidades de mampostería Los ladrillos de la vivienda son de tipo tubular (pandereta) que según la Norma Peruana no está permitido por tratarse de muros portantes (Norma E.070, 2006); además presentan fisuras y grietas considerables. Por lo tanto, la vivienda

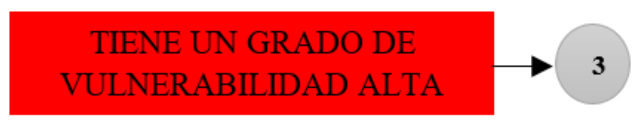

C) Calidad de los materiales

Las vigas y columnas se encuentran expuestas. Y los elementos de confinamiento en concreto reforzado, tienen estribos abundantes de $3 / 8$ ". Por lo tanto, la vivienda

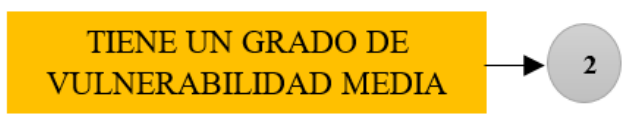

Parámetro 03: Aspectos Estructurales

A) Muros confinados y reforzados

Todos los muros de la vivienda están confinados con vigas y columnas de concreto reforzado.

La distancia máxima entre ejes de columnas es de $4.45 \mathrm{~m}$., por lo tanto, se encuentra dentro del rango que señala el RNE (Norma E.070, 2006). Los elementos de confinamiento tienen refuerzo tanto longitudinal como transversal y está adecuadamente dispuesto. Por lo tanto, la vivienda 


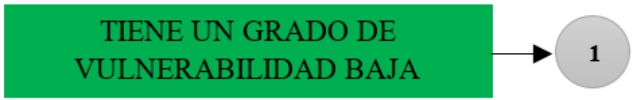

B) Detalles de columnas y vigas de confinamiento Las columnas y vigas tienen $20 \mathrm{~cm}$ de espesor y 400 $\mathrm{cm} 2$ de área transversal, con refuerzos anclados en sus extremos y a los elementos de la cimentación, con 4 barras No 4 longitudinales y estribos de $\mathrm{N}^{\circ} 3$. Por lo tanto, la vivienda

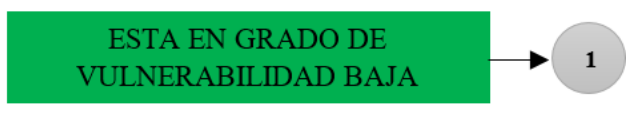

C) Vigas de amarre o corona

La vivienda cuenta con viga de amarre o corona de concreto armado en sus muros. Por lo tanto, la vivienda

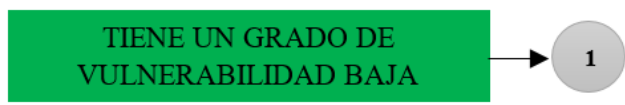

D) Características de las aberturas

De la vivienda, se calcula:

- Área de las aberturas en relación al área total del muro.

En la dirección " $\mathrm{X}$ "

(Área de las aberturas en "X")/(Área total del muro en "X") $=2.40 / 12.30=0.20=20 \%$

$E l$ área de las aberturas en " $X$ " viene a ser el $20 \%$ del área total de muro en " $X$ "

- Longitud total de las aberturas en relación a la longitud total del muro.

En la dirección " $\mathrm{X}$ "

(Long. de las aberturas en "X")/(Long. total del muro en "X")=3.00/6.15 = $0.49=49 \%$

La longitud de las aberturas en " $X$ " viene a ser el $49 \%$ de la longitud total de muro en la dirección " $X$ ".

- Las aberturas en los muros totalizan menos del $35 \%$ del área total del muro.

- La longitud total de aberturas en el muro corresponde a menos del $50 \%$ de la longitud total del muro.

- Existe una distancia desde el borde del muro hasta la abertura adyacente de $0.25 \mathrm{~m}$.

- La distancia entre aberturas es menor al $0.50 \mathrm{~m}$. Por lo tanto, la vivienda

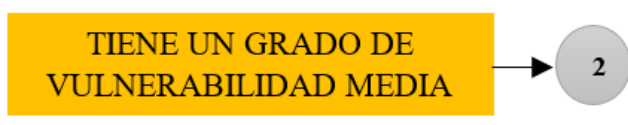

E) Entrepiso

La vivienda es de 2 niveles. Con un entrepiso liviano construido de vigas y piso de madera. La madera se apoya de manera adecuada a los muros de soporte. Por lo tanto, la vivienda

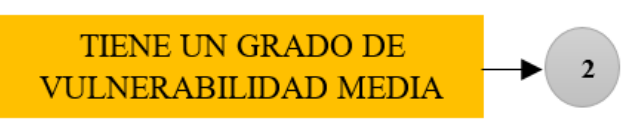

F) Amarre de cubiertas

La vivienda usó tornillos para fijar la cubierta liviana a los muros confinados. Por lo tanto, la vivienda

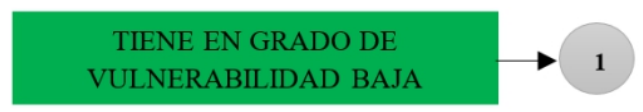

Parámetro 04: Cimentación

El método nos dice: "Si no viste el proceso de construcción de la vivienda, considera que tiene vulnerabilidad media (AIS, 2001). Por lo tanto, la vivienda

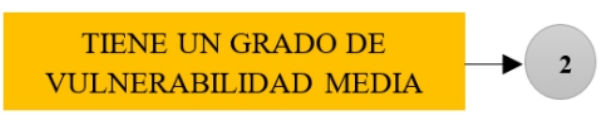

Parámetro 05: Suelos

El suelo fue un relleno mal compactado lo que le hace ser blando. Por lo tanto, la vivienda

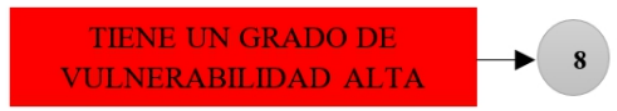

Parámetro 06: Entorno

La vivienda se encuentra ubicada en una topografía con una inclinación de $27^{\circ}$ con la horizontal. Por lo tanto,

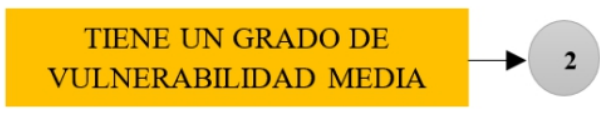

1. Calificación global de la vivienda

Tabla 1. Calificación Global de la vivienda $\mathrm{N}^{\circ} 21$

\begin{tabular}{|c|r|r|r|}
\hline \multirow{2}{*}{ COMPONENTE } & \multicolumn{3}{|c|}{ VULNERABILIDAD } \\
\cline { 2 - 5 } & BAJA & MEDIA & ALTA \\
\hline 1) ASPECTOS GEOMÉTRICOS & & & \\
\hline a) Irregularidad en planta de la edificación & 1 & & \\
\hline b) Cantidad de muros en las dos direcciones & 1 & & \\
\hline c) Irregularidad en altura & & & \\
\hline 2) ASPECTOS CONSTRUCTIVOS & & 2 & \\
\hline a) Calidad de las juntas de pega del mortero & & & 3 \\
\hline b) Tipo y disposición de las unidades de mampostería & & 2 & \\
\hline c) Calidad de los materiales & & & \\
\hline 3) ASPECTOS ESTRUCTURALES & 1 & & \\
\hline a) Muros confinados y reforzados & 1 & & \\
\hline b) Detalles de columnas y vigas de confinamiento & 1 & & \\
\hline c) Vigas de amarre o corona & & 2 & \\
\hline d) Caracteristicas de las aberturas & & 2 & \\
\hline e) Entrepiso & & & \\
\hline f) Amarre de cubiertas & & & \\
\hline 4) CIMENTACIÓN & & & \\
\hline 5) SUELOS & & & \\
\hline 6) ENTORNO & & & \\
\hline CALIFICACIÓN GLOBAL & & & \\
\hline
\end{tabular}

\section{Análisis de datos}

Los datos obtenidos por medio de la Ficha de Recolección de Datos fueron analizados mediante los parámetros que señala el Método (AIS), aplicando las Normas técnicas peruanas; Y su calificativo de grado de vulnerabilidad de cada parámetro de vivienda fue procesado en hojas de cálculo del Software Microsoft Excel. Obteniendo resultados satisfactorios que demuestran que la hipótesis de la investigación es verdadera. 


\section{RESULTADOS}

Tabla 2. Grado de vulnerabilidad sísmica

\begin{tabular}{|c|c|c|c|}
\hline $\begin{array}{l}\mathrm{N}^{\circ} \text { de } \\
\text { ivienda }\end{array}$ & Características relevantes & Calificación & $\begin{array}{c}\text { Grado de } \\
\text { vulnerabilidad sísmica }\end{array}$ \\
\hline 1 & Muros de adobe sin arriostrar & 35 & ALTA \\
\hline 2 & Muro de ladrillo pandereta & 25 & MEDIA \\
\hline 3 & Muros de adobe sin arriostrar & 33 & ALTA \\
\hline 4 & Muros de adobe sin arriostrar & 41 & ALTA \\
\hline 5 & Muros de adobe sin arriostrar & 59 & ALTA \\
\hline 6 & Muros de adobe sin arriostrar & 27 & MEDIA \\
\hline 7 & Muros de adobe sin arriostrar & 38 & ALTA \\
\hline 8 & Muros de adobe sin arriostrar & 40 & ALTA \\
\hline 9 & Muros de adobe sin arriostrar & 39 & ALTA \\
\hline 10 & Muros de adobe sin arriostrar & 49 & ALTA \\
\hline 11 & Muros de adobe sin arriostrar & 49 & ALTA \\
\hline 12 & Muros de adobe sin arriostrar & 48 & ALTA \\
\hline 13 & Muros de adobe sin arriostrar & 44 & ALTA \\
\hline 14 & Muro de ladrillo pandereta & 37 & ALTA \\
\hline 15 & Muro de ladrillo pandereta & 45 & ALTA \\
\hline 16 & Muros de adobe arriostrado & 28 & MEDIA \\
\hline 17 & Muro de ladrillo pandereta & 24 & MEDIA \\
\hline 18 & Muros de adobe arriostrado & 31 & MEDIA \\
\hline 19 & Muros de adobe sin arriostrar & 32 & ALTA \\
\hline 20 & Muro de ladrillo pandereta & 46 & ALTA \\
\hline 21 & Muro de ladrillo pandereta & 30 & MEDIA \\
\hline 22 & Muros de adobe sin arriostrar & 38 & ALTA \\
\hline 23 & Muro de ladrillo pandereta & 31 & MEDIA \\
\hline 24 & Muro de ladrillo pandereta & 29 & MEDIA \\
\hline 25 & Muros de adobe $\sin$ arriostrar & 39 & ALTA \\
\hline 26 & Muros de adobe sin arriostrar & 39 & ALTA \\
\hline 27 & Muro de ladrillo pandereta & 20 & MEDIA \\
\hline 28 & Muro de ladrillo pandereta & 19 & BAJA \\
\hline 29 & Muro de ladrillo pandereta & 26 & MEDIA \\
\hline 30 & Muro de ladrillo pandereta & 20 & MEDIA \\
\hline
\end{tabular}

Resumen del grado de vulnerabilidad sísmica

Tabla 3. Resumen del grado de vulnerabilidad sísmica

\begin{tabular}{ccc}
\hline $\begin{array}{c}\text { RESUMEN GADO DE VULNERABILIDAD } \\
\text { SÍSMIA DE LAS VIVIENDAS }\end{array}$ \\
$\begin{array}{c}\text { GRADO DE } \\
\text { VULNERABILIDAD }\end{array}$ & TOTAL & TOTAL (\%) \\
\hline BAJA & 1 & $3.33 \%$ \\
\hline MEDIA & 11 & $36.67 \%$ \\
\hline ALTA & 18 & $60 \%$ \\
\hline TOTAL & $\mathbf{3 0}$ & $\mathbf{1 0 0 \%}$ \\
\hline
\end{tabular}

\section{DISCUSIÓN}

El estudio de la vulnerabilidad sísmica de las viviendas informales del AA. HH. San Carlos de Murcia, determinó que el 3.33\% de las viviendas analizadas presentan un grado de vulnerabilidad sísmica baja, el $36.67 \%$ de las viviendas analizadas presentan un grado de vulnerabilidad media y el $60 \%$ de las viviendas analizadas presentan un grado de vulnerabilidad sísmica alta. Esto confirma que la hipótesis planteada, en donde se afirma que el grado de vulnerabilidad sísmica de las viviendas de este sector es alto, es verdadera.

La investigación de pregrado "Análisis de la vulnerabilidad sísmica de las viviendas informales en la ciudad de Trujillo" (Laucata, 2013), menciona que, de las 30 viviendas analizadas de la ciudad de Trujillo, el 7\% presentó una vulnerabilidad sísmica baja, el $10 \%$ presentó una vulnerabilidad sísmica media y el $83 \%$ presentó una vulnerabilidad sísmica alta. Similarmente al resultado de la presente investigación, donde la mayor parte de las viviendas se encuentran en un grado de vulnerabilidad sísmica alta, la diferencia de porcentajes se debe a que Laucata (2013) se basó en investigar solamente viviendas de albañilería confinada, contrario a la presente investigación que se basó también en las viviendas de adobe, además eligió zonas de estudio donde su tipología y topografía de suelo reflejaban 
mayor riesgo y distinto al suelo con el que disponemos en esta presente investigación.

Si comparamos la investigación de pregrado "Evaluación de la vulnerabilidad sísmica en viviendas de albañilería confinada del Asentamiento Humano San Marcos de Ate, Santa Anita, 2017" (Rojas, 2017) indica que, de las 15 viviendas analizadas, el $6.67 \%$ presentó una vulnerabilidad sísmica baja, el $33.33 \%$ presentó una vulnerabilidad sísmica media y el $60.00 \%$ presentó una vulnerabilidad sísmica alta; caso que en la presente investigación se cuenta con el mismo porcentaje de grado de vulnerabilidad alta, pero con diferente porcentaje en la vulnerabilidad sísmica media y baja, debido a que Rojas (2017) analiza viviendas de albañilería confinada con y sin asesoría técnica, resultando que las viviendas que contaron con asesoría técnica fueron las mejor construidas y se identificaron con una vulnerabilidad media o baja.

La investigación de pregrado "Evaluación de la vulnerabilidad sísmica de las edificaciones comunes en la ciudad de Pimentel" (Mesta, 2014), señala que se evaluó 3026 viviendas, obteniendo que: el 20.8\% de las viviendas analizadas presentaron un grado de vulnerabilidad sísmica baja, el $33.5 \%$ presentaron un grado de vulnerabilidad sísmica media y el $45.7 \%$ presentaron un grado de vulnerabilidad sísmica alta. Al compararla con la presente investigación los resultados son similares, diferenciándose en los porcentajes obtenidos, esto debido que Mesta (2014) analizó viviendas de adobe, albañilería y concreto armado, que recibieron asesoría técnica, así como también las que no lo hicieron; una vivienda construida con asesoría técnica y de concreto armado se va encontrar en un grado de vulnerabilidad sísmica menor que una que fue construida a base de adobe.

En la investigación de pregrado "Diagnóstico de vulnerabilidad estructural para verificar viviendas, urbanización Carlos Stein Chávez Primera Etapa, José Leonardo Ortiz, Lambayeque-2016" (Díaz, 2016), señala que evaluó 15 viviendas, resultando que: el $40 \%$ presentaron una vulnerabilidad sísmica baja, 53.33\% presentaron una vulnerabilidad sísmica media y el $6.67 \%$ presentaron una vulnerabilidad sísmica alta. En cambio, en los resultados de la presente investigación la mayor parte de viviendas se encuentran en un grado de vulnerabilidad alta, utilizando el mismo método de la Asociación Colombiana de Ingeniería Sísmica. La diferencia se debe a que Díaz (2016) solo se basó en analizar viviendas de mampostería confinada autoconstruidas como las que recibieron asesoría técnica para su construcción, ubicadas en la ciudad de Chiclayo, donde tipología y topografía del suelo es diferente.

En el caso de la investigación de pregrado "Estimación del riesgo sísmico de las viviendas autoconstruidas en el barrio Santa Isabel, Chachapoyas, Amazonas, 2016". (Oc, 2017), se señala que, de las 61 viviendas evaluadas, el $11.5 \%$ se encontraron con una vulnerabilidad sísmica baja; el $23 \%$ se encontraron con una vulnerabilidad sísmica media; el 59\% se encontraron con una vulnerabilidad sísmica alta y el $3.3 \%$ se encontraron con una vulnerabilidad sísmica muy alta. La investigación de Oc. (2017), concuerda con la presente investigación y es válida su comparación ya que se realizó en la misma ciudad y teniendo en cuenta, también, viviendas construidas a base de adobe y de ladrillo. La diferencia de porcentajes en los resultados obtenidos se debe a que Oc. (2017) evaluó la vulnerabilidad sísmica de las viviendas considerando 4 indicadores de calificación: baja, media, alta y muy alta.

En la investigación de pregrado "Evaluación de la vulnerabilidad sísmica de las viviendas autoconstruidas de dos pisos de albañilería confinada en el Asentamiento Humano Pedro Castro Alva, Chachapoyas, 2017" (Linares, 2019), se obtuvo, que de las 48 viviendas analizadas: el $0 \%$ presentaron un nivel de vulnerabilidad sísmica bajo, el $68.8 \%$ presentaron un nivel de vulnerabilidad sísmica moderado, el $25.0 \%$ presentaron un nivel de vulnerabilidad sísmica alto y el $6.2 \%$ presentaron un nivel de vulnerabilidad sísmica muy alto. Se compara la investigación con la presente y se analiza que la diferencia en los resultados se debe a que Linares (2019) solo se basó en el análisis de las viviendas de dos pisos de albañilería confinada, en cambio la investigación actual se basó en viviendas de adobe y ladrillo de uno y dos pisos; en los resultados de la presente investigación (Tabla 2) se observa que las viviendas de albañilería confinada son las que se encuentran en un grado de vulnerabilidad baja o media, concordando así con la investigación de Linares (2019).

\section{CONCLUSIONES}

El grado de vulnerabilidad sísmica de las viviendas informales en el AA. HH. San Carlos de Murcia es alto, ya que el $60 \%$ del total de las viviendas estudiadas se encontraron con un grado de vulnerabilidad sísmica alta, el 3.33\% de las viviendas analizadas presentan un grado de vulnerabilidad sísmica baja y el $36.67 \%$ de las viviendas analizadas presentan un grado de vulnerabilidad media, comprobando así la hipótesis de esta investigación. Las 30 viviendas (17 de adobe y 13 de albañilería), fueron seleccionadas teniendo en cuenta su ubicación y topografía.

La vivienda que se encuentra en mayor riesgo es la $\mathrm{N}^{\circ}$ 05 (puntos: 59, Tabla 2), categorizada en un grado de vulnerabilidad sísmica alta. Esta vivienda de adobe, según la evaluación que se hizo no cuenta con muros 
confinados y bien distribuidos en las principales direcciones " $\mathrm{X}$ " e "Y"; además de encontrarse en un mal estado actual (fisuras, grietas), etc. y de ubicarse en una zona accidentada.

La vivienda que se encuentra en menor riesgo es la $\mathrm{N}^{\circ} 28$ (puntos: 19, Tabla 2), categorizada como vulnerablemente baja, por haberse construido sobre un suelo de fundación adecuado con materiales aceptables, y consideraciones que son aceptables para el R.N.E.

\section{REFERENCIAS BIBLIOGRÁFICAS}

AIS. (2001). Asociación Colombiana de Ingeniería Sísmica. Manual de construcción, evaluación y rehabilitación sismo resistente de viviendas de mampostería. Colombia: Editorial La Red.

Aragón, J., Flores, L. y López, O. (2011). Manual del Formato de captura de datos para evaluación estructural. Red Nacional de Evaluadores. Colombia: CENAPRED.

Díaz, V. R. (2016). Diagnóstico de vulnerabilidad estructural para verificar viviendas, urbanización Carlos Stein Chávez Primera Etapa, José Leonardo Ortiz, Lambayeque2016. Tesis para optar el título de Ingeniero Civil. Universidad César Vallejo, Chiclayo.

Laucata, J. E. (2013). Análisis de la vulnerabilidad sísmica de las viviendas informales en la ciudad de Trujillo. Tesis para optar el título de Ingeniero Civil. Pontificia Universidad Católica del Perú, Lima.

Linares, N. F. (2019). Evaluación de la vulnerabilidad sísmica de las viviendas autoconstruidas de dos pisos de albañilería confinada en el Asentamiento Humano Pedro Castro Alva, Chachapoyas, 2017. Tesis para obtener el título profesional de Ingeniero Civil. Universidad Nacional Toribio Rodríguez de Mendoza de Amazonas, Chachapoyas.

Mesta, C. A. (2014). Evaluación de la vulnerabilidad sísmica de las edificaciones comunes en la ciudad de Pimentel. Tesis para optar el título profesional de Ingeniero Civil. Universidad de San Martín de Porres, Chiclayo.

Norma. E.030. (2016). Reglamento Nacional de Edificaciones - Diseño sismorresistente. Lima: ICG.

Norma. E.070. (2006). Reglamento Nacional de Edificaciones - Albañilería. Lima: ICG.
Norma. E.080. (2017). Reglamento Nacional de Edificaciones - Diseño y construcción con tierra reforzada. Lima: ICG.

Oc, J. (2017). Estimación del riesgo sísmico de las viviendas autoconstruidas en el barrio Santa Isabel, Chachapoyas, Amazonas, 2016. Tesis para obtener el título profesional de Ingeniero Civil. Universidad Nacional Toribio Rodríguez de Mendoza de Amazonas, Chachapoyas.

Rojas, E. (2017). Evaluación de la vulnerabilidad sísmica en viviendas de albañilería confinada del Asentamiento Humano San Marcos de Ate, Santa Anita, 2017. Tesis para obtener el título profesional de Ingeniero Civil. Universidad César Vallejo, Lima. 